\title{
Novel magnetic resonance imaging marker of diffuse myocardial fibrosis in hypertensive heart disease: the role of transcytolemmal water- exchange
}

\author{
Otavio R Coelho-Filho ${ }^{1,2^{*}}$, Richard N Mitchell ${ }^{1}$, Heitor Moreno ${ }^{1}$, Raymond Kwong ${ }^{2}$, Michael Jerosch-Herold ${ }^{3}$ \\ From 15th Annual SCMR Scientific Sessions \\ Orlando, FL, USA. 2-5 February 2012
}

\section{Summary}

Transcytolemal water exchange and its effect on myocardial T1 relaxation can, if neglected, lead to a significant underestimate of the myocardial extracellular volume fraction, a novel marker of diffuse fibrosis.

\section{Background}

LGE may fail to detect diffuse fibrosis in several cardiac conditions. A novel approach uses the myocardial extracellular volume-fraction (MECVF), measured as distribution volume of a gadolinium constrast, as a marker of extracellular expansion. Previous studies have assumed the fast-exchange (FX) limit for the transcytolemal waterexchange, yet the administration of an extracellular-agent can create significant transcytolemal T1-differences, and cause an underestimation of extracellular volume fraction under the FX assumption. We hypothesized that the quantitative measure of MECVF with using a 2-site $\mathrm{H}$-exchange model (2SX-model) correlates positively with the extracellular volume fraction, while the FX approach underestimates extra-cellular matrix expansion in a rodent model of hypertensive heart disease and diffuse myocardial fibrosis created by administration of L-NAME.

\section{Methods}

L-NAME $(3 \mathrm{mg} / \mathrm{ml})$ or placebo was administered respectively to $22(\mathrm{bw}=36.9 \pm 2.3 \mathrm{~g})$ and $15(\mathrm{bw}=37.6 \pm 2.5 \mathrm{~g})$ wildtype mice. Animals were imaged at baseline and 7weeks after treatment on a 4.7T small-animal MRI-system. T1(\# of T1's $>5 /$ mouse) was quantified with a

${ }^{1}$ Medicine, State University of Campinas, Campinas, Brazil Full list of author information is available at the end of the article modified Look-Locker gradient-echo-cine technique, before and after fractionated Gd-DPTA administration (mean max. R1 in blood post-contrast $=5.0 \pm 2.261 / \mathrm{s}$ ). MECVF obtained from the T1 measurements with the 2SX and FX-models, and by using blood hematocrit to adjust the partition coefficient. Connective tissue volume fraction (CTVF) was measured using Masson's trichrome.

\section{Results}

L-NAME-treated animals demonstrated hypertrophy (weight-indexed LVmass $4.1 \pm 0.4$ vs. $2.2 \pm 0.3 \mu \mathrm{g} / \mathrm{g}$, $\mathrm{p}<0.001)$ and increased CTVF $(8.6 \% \pm 1.5$ vs. $2.58 \% \pm 0.6$, $\mathrm{P}<0.001)$ as compared to controls. MECVF was substantially higher in L-NAME-treated animals $(0.43 \pm 0.09$ vs, $0.26 \pm 0.03, \mathrm{p}<0.001$ with $2 \mathrm{SX}$-model; $0.20 \pm 0.03$ vs. 0.20 $\pm 0.05, \mathrm{p}=0.82$ without TWE with FX assumption). MECVF from 2SX-model showed a good correlation with CTVF and weight-indexed LVmass (both $r=0.8$, $\mathrm{p}<0.0001$ ), while MECVF from FX-model did not correlate significantly with $\operatorname{CTVF}(\mathrm{p}=0.44)$ and LVmass $(\mathrm{p}=0.80)$ (fig-1). MECVF from the 2SX model also correlated with the LVEF at 7 -weeks $(r=0.48, p=0.01)$. BlandAltman analysis demonstrated that neglect of the transcytolemal water exchange causes a significant underestimate MECVF expansion(fig-2), which worsens with extracellular matrix expansion and LVH.

\section{Conclusions}

CMR MECVF quantification provides a robust measure of myocardial extracellular matrix expansion and interstitial fibrosis, though any break-down of the FX assumption for transcytolemmal exchange can result in a significant 

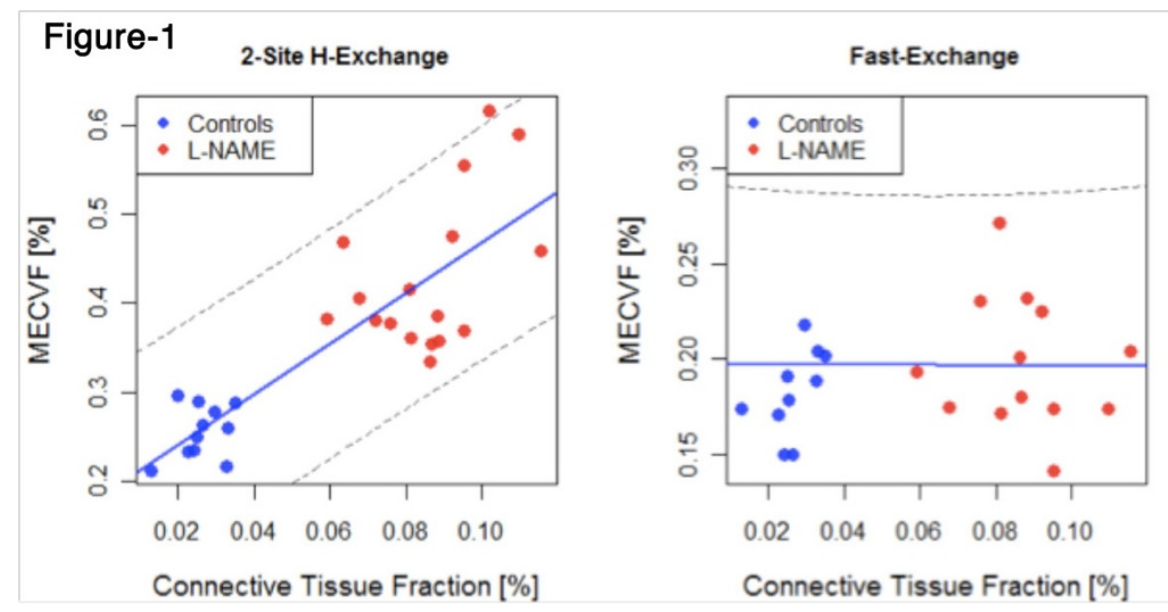

Figure 1 Correlation: MECVF (2-site H-exchange and Fast-exchange) and connective tissue volume fraction

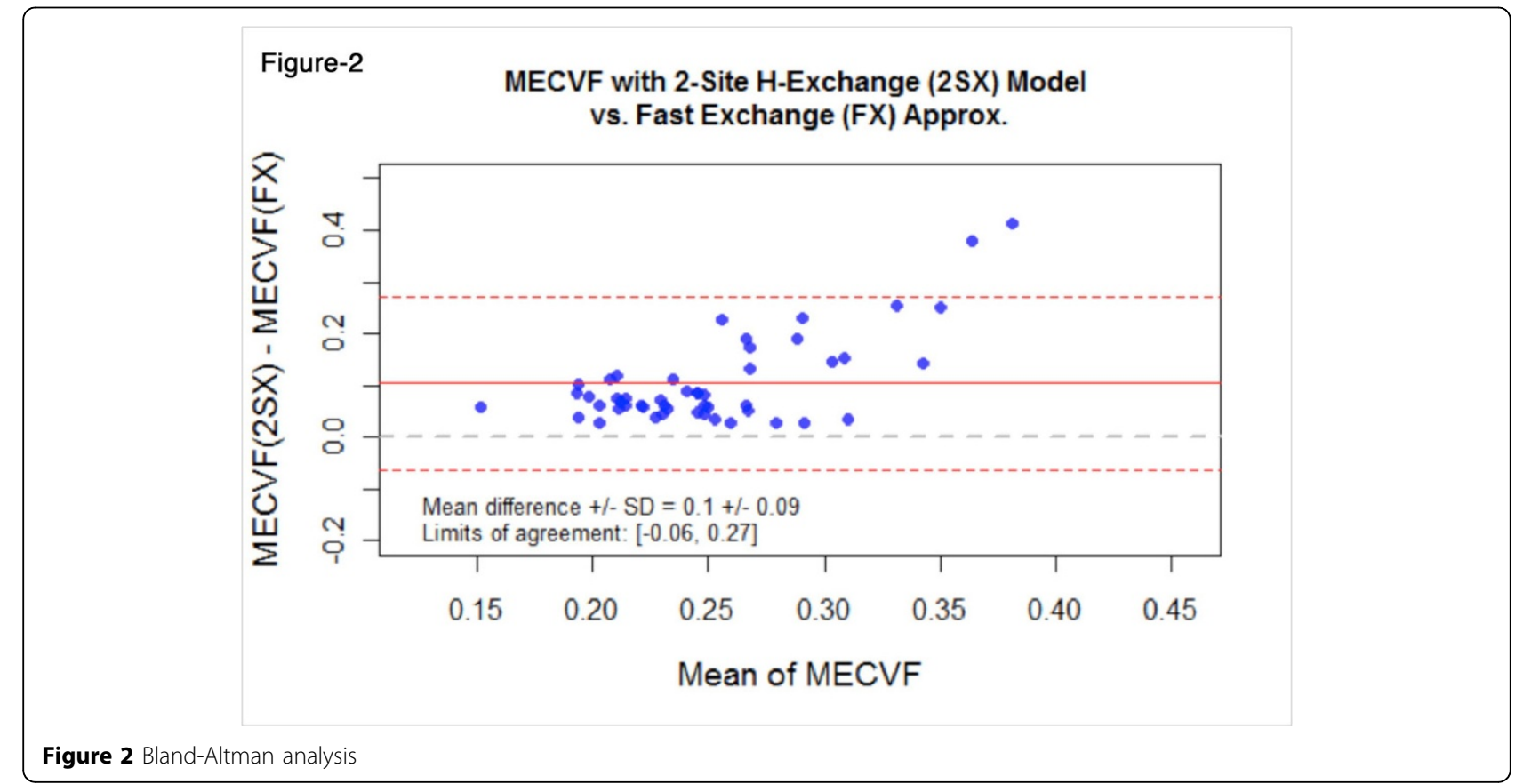

underestimation of MECVF. Importantly, underestimates of MECVF due to the FX assumption depend on the degree of cell-hypertrophy, and the maximum $\mathrm{T} 1$ in the blood pool. A break-down of the FX assumption cannot be detected with protocols limited to a pair of pre/postcontrast T1 measurements. A generalization of the model for determination of MECVF brings important benefits for an early detection of diffuse fibrosis.

\section{Funding}

Supported by the American Heart Association (AHA 11POST5550053) and the National Institutes of Health/ NHLBI (1R01HL090634-01A1).

\section{Author details}

${ }^{1}$ Medicine, State University of Campinas, Campinas, Brazil. ${ }^{2}$ Medicine, Brigham and Women's Hospital, Boston, MA, USA. ${ }^{3}$ Radiology, Brigham and Women's Hospital, Boston, MA, USA.

Published: 1 February 2012

doi:10.1186/1532-429X-14-S1-0115

Cite this article as: Coelho-Filho et al:: Novel magnetic resonance imaging marker of diffuse myocardial fibrosis in hypertensive heart disease: the role of transcytolemmal water-exchange. Journal of Cardiovascular Magnetic Resonance 2012 14(Suppl 1):O115. 